\title{
HOMOLOGY IN VARIETIES OF GROUPS. I
}

\author{
BY \\ C. R. LEEDHAM-GREEN
}

\begin{abstract}
Well-known techniques allow one to construct a (co-) homology theory relative to a variety. After two paragraphs which discuss the modules to be considered and the construction of the (co-) homology groups, we come to our main homological result, namely that the theory is not always equivalent to a Tor or Ext. In the fourth paragraph we prove our main group-theoretic result; two covering groups of a finite group generate the same variety "up to exponent". Finally we produce a restricted version of the Künneth formula.
\end{abstract}

Introduction. Well-known techniques allow one to construct a (co-) homology theory relative to a variety. After two paragraphs which discuss the modules to be considered and the construction of the (co-) homology groups, we come to our main homological result, namely that the theory is not always equivalent to a Tor or Ext. In the fourth paragraph we prove our main group-theoretic result; two covering groups of a finite group generate the same variety "up to exponent". Finally we produce a restricted version of the Künneth formula.

It is intended in later papers to consider the problem raised in paragraph 3 for modules with trivial action, to demonstrate the wild behaviour of the higher homology groups, and to examine a theory which arises when one considers twosided modules.

This paper has its genesis in some unpublished work of Peter M. Neumann and myself.

Preliminaries. If $\mathscr{C}$ is a category, $|\mathscr{C}|$ denotes the class of objects in $\mathscr{C}$, and if $A, B \in|\mathscr{C}|$, then $\mathscr{C}(A, B)$ denotes the set of morphisms of $A$ into $B$. If $\Pi \in|\mathscr{C}|$, the comma category $(\mathscr{C}, \Pi)$ has as objects morphisms in $\mathscr{C}$ with range $\Pi$ and as morphisms the usual commutative triangles. We shall usually write objects and morphisms in $(\mathscr{C}, \Pi)$ as objects and morphisms in $\mathscr{C}$, the morphisms into $\Pi$ being understood. In particular, $\Pi$ denotes the terminal object $1_{\Pi}$ of $(\mathscr{C}, \Pi)$. $\mathfrak{B}$ will be a variety regarded as a full subcategory of the category of all groups, and $\Pi$ a group in $\mathfrak{B}$. If $A$ is a $\Pi$-module and $\Gamma \rightarrow \Pi \in|(\mathfrak{B}, \Pi)|$, then $A$ will be regarded as a $\Gamma$-module by pullback. Whether $A$ is a left or right module will often be left

Received by the editors September 2, 1970.

AMS 1970 subject classifications. Primary 18C15, 18G15, 18H40, 20E10, 20J05; Secondary $18 \mathrm{G} 10,18 \mathrm{H} 10,18 \mathrm{H} 15$.

Key words and phrases. Variety of groups, (co-) homology of groups, triple homology, Kan extension, Rinehart's exact sequence, Fox derivative, group extension, Schur multiplier, covering group, Künneth formula. 
deliberately ambiguous; one is to understand left module in the case of homology and right module in the case of cohomology. $Z \Gamma$ and $I \Gamma$ denote respectively the integral group ring and augmentation ideal of the group $\Gamma$. If $A$ is a $\Pi$-module, Der $(\Gamma, A)$ and Diff $(\Gamma, A)$ denote the group $\operatorname{Hom}_{z \Gamma}(I \Gamma, A)$ of right derivations of $\Gamma$ into $A$, and $\Gamma \otimes_{z \Gamma} A$ respectively, $A$ being understood according to our convention as a right module in the former case and a left module in the latter. $[x, y]$ denotes the commutator $x^{-1} y^{-1} x y$ and higher commutators are left normed. $x^{y}=y^{-1} x y$. $\zeta \Gamma$ denotes the centre of the group $\Gamma, \Gamma^{\prime}=\Gamma_{(2)}$ its derived group, and $\Gamma_{(n)}$ the $n$th term of the lower central series.

1. Modules in a variety. If $F$ is the free group on a set $\mathfrak{x}$, and if $x \in \mathfrak{x}$, then $\partial / \partial x$ is the right derivation of $F$ into $Z F$ which sends $x$ to 1 and every other element of $x$ to 0 . If $w \in F$, then $\partial w / \partial x$ is the Fox derivative of $w$ with respect to $x$ (see Fox [8]). The variety $\mathfrak{B}$ determines a normal subgroup $\mathfrak{B} \mathfrak{x}$ of $F$. Every homomorphism of $F$ into $\Pi$ determines a homomorphism of $Z F$ into $Z \Pi$. Let $\mathfrak{B} \Pi$ be the factor ring of $Z \Pi$ by the ideal generated by the image under all such homomorphisms of the Fox derivatives of every element of $\mathfrak{B x}$. It is sufficient to replace $\mathfrak{B} \mathfrak{x}$ by any set of words which define $\mathfrak{B}$ (Knopfmacher [10]). Moreover, if $\mathfrak{B}$ is determined by its $n$-letter words, then $F$ can be replaced by the free group of rank $n$.

THEOREM 1.1 (KNOPfMACHER [10]). Let $A$ be a П-module. Then the split extension $A \Pi$ lies in $\mathfrak{B}$ if and only if $A$ is a $\mathfrak{B} \Pi$-module.

An abelian group in $(\mathfrak{B}, \Pi)$ is a split extension $A \Pi$ with its canonical projection on $\Pi ; A$ a $\Pi$-module such that $A \Pi$ lies in $\mathfrak{B}$. This is clearly equivalent to the usual definition. Thus the full subcategory of $(\mathfrak{B}, \Pi)$ whose objects are the abelian groups is isomorphic to $\mathscr{M} \mathfrak{B}_{\Pi}$, the category of right $\mathfrak{B} \Pi$-modules. We use the obvious isomorphism to confuse these categories. If $\mathfrak{B}$ is the variety of all groups, we write $\mathscr{M}_{\Pi}$ for $\mathscr{M} \mathfrak{B}_{\Pi}$.

We now construct a left adjoint to the inclusion of $\mathscr{M}_{\mathfrak{B}_{\Pi}}$ into $(\mathfrak{B}, \Pi)$. Let $\Gamma \rightarrow \Pi \in|(\mathfrak{B}, \Pi)|$, and $A \in\left|\mathscr{M}_{\mathfrak{B}_{\Pi}}\right|$. Then $(\mathfrak{B}, \Pi)(\Gamma, A)=\operatorname{Der}(\Gamma, A)=\mathscr{M}_{\Gamma}(I \Gamma, A)$ $=\mathscr{M}_{\Pi}\left(I \Gamma \otimes_{z \Gamma} Z \Pi, A\right)=\mathscr{M}_{\Pi}\left(I \Gamma \otimes_{z \Gamma} \mathfrak{B} \Pi, A\right)$. Thus if we put $D \Gamma=I \Gamma \otimes_{z \Gamma} \mathfrak{B} \Pi$ then $D$ is the desired adjoint.

LEMMA 1.2. If $F$ is $\mathfrak{B}$-freely generated by $\mathfrak{x}$, then $D F$ is the free $\mathfrak{B} \Pi$-module on $\{(1-x) \otimes 1 \mid x \in \mathfrak{x}\}$.

Proof. If $A$ is any $\mathfrak{B} \Pi$-module, then $\operatorname{Der}(F, A)$ is the product of $\mathfrak{x}$ copies of $A$, the projection on the $x$ th factor being given by $\delta \mapsto x \delta$. But there is an isomorphism $\theta: \operatorname{Der}(F, A) \rightarrow \mathscr{M}_{\mathfrak{B}_{\mathrm{\Pi}}}(D F, A)$, natural in $A$, given by $((1-x) \otimes 1) \delta \theta=x \delta$. Hence $D F$ is freely generated by $\{(1-x) \otimes 1 \mid x \in \mathfrak{x}\}$.

We end this section with a result we need later, which is stated (without proof) in [10]. $\mathfrak{R}_{c}$ denotes the variety of groups nilpotent of class at most $c, c \geqq 1$.

Proposition 1.3. If $\Pi \in \mathfrak{R}_{c}$, then $\mathfrak{R}_{c} \Pi=Z \Pi /(I \Pi)^{c}$. 
Proof. Let $F$ be the absolutely free group on $x_{1}, x_{2}, \ldots$ It is easy to prove that the Fox derivatives of any element of $F_{(c+1)}$ are in $(I F)^{c}$. Hence if $\theta: F \rightarrow \Pi$ is any homomorphism, the induced homomorphism of $Z F$ into $Z \Pi$ maps these Fox derivatives into $(I \Pi)^{c}$. Conversely, a simple calculation shows

$$
\begin{aligned}
\partial\left[x_{1}, x_{2}, \ldots, x_{c+1}\right] / \partial x_{1}= & x_{2} \cdots x_{c+1}\left(1-\left(x_{2}^{-1}\right)^{x_{1} \ldots x_{c+1}}\right)\left(1-\left(x_{3}^{-1}\right)^{\left[x_{1}, x_{2}\right] x_{3} \ldots x_{c+1}}\right) \\
& \cdots\left(1-\left(x_{i}^{-1}\right)^{\left[x_{1}, \ldots, x_{i-1}\right] x_{i} \ldots x_{c+1}}\right) \cdots\left(1-\left(x_{c+1}^{-1}\right)^{\left[x_{1}, \ldots, x_{c}\right] x_{c+1}}\right) .
\end{aligned}
$$

We prove that

$$
\left\{\left(x_{i}^{-1}\right)^{\left[x_{1}, \ldots, x_{i-1}\right] x_{i} \ldots x_{c+1}} \mid i=2, \ldots, c+1\right\}
$$

is part of a free generating set of $F$. Conjugating by $\left(x_{1} \cdots x_{c+1}\right)^{-1}$, it is enough to prove that

$$
T=\left\{\left(x_{i}^{-1}\right)^{\left[x_{1}, \ldots, x_{i-1}\right] x_{i-1}^{-1} \ldots x_{i}^{-1}} \mid i=2, \ldots, c+1\right\}
$$

is part of a free generating set of $F$. But $T \cup\left\{x_{1}, x_{c+2}, x_{c+3}, \ldots\right\}$ clearly generates $F$ freely. Thus if $a_{1}, a_{2}, \ldots, a_{c}$ is any sequence of $c$ elements of $\Pi$ there is a homomorphism $\theta: F \rightarrow \Pi$ mapping $\left(x_{i}^{-1}\right)^{\left[x_{1}, \ldots, x_{i-1}\right] x_{i} \ldots x_{c+1}}$ to $a_{i-1} ; i=2, \ldots, c+1$. So $u\left(1-a_{1}\right) \cdots\left(1-a_{c}\right)$ is the image of a Fox derivative of an element of $F_{(c+1)}$, for some $u \in \Pi$; hence $\left(1-a_{1}\right) \cdots\left(1-a_{c}\right)$ is in the ideal generated by these images.

In particular if $c=1, \mathfrak{R}_{c}$ is the ring of integers and $D$ is the identity functor, as can be seen directly.

There is one other case which will be of importance later. If $\mathfrak{A}$ is the variety of abelian groups, the product variety $\mathfrak{A} \mathfrak{B}$ consists of those groups which can be expressed as an extension of an abelian group by a group in $\mathfrak{B}$. Theorem 1.1 now gives

Proposition 1.4. If $\mathfrak{B}$ contains $\mathfrak{A}$ var $\Pi$, then $\mathfrak{B} \Pi=Z \Pi$.

More generally,

Proposition 1.5. If $\mathfrak{B}$ contains $\mathfrak{A}$ var $\Pi$ and $\mathfrak{U}$ contains $\Pi$, then $(\mathfrak{B} \cap \mathfrak{u}) \Pi=\mathfrak{u} \Pi$.

2. (Co-) homology. Let $\mathscr{P}$ denote the full subcategory of $\mathfrak{B}$ whose objects are the $\mathfrak{B}$-free groups, and $(\mathscr{P}, \Pi)$ denote the corresponding subcategory of $(\mathfrak{B}, \Pi)$. If $T$ is a functor from $(\mathfrak{B}, \Pi)$ or $(\mathscr{P}, \Pi)$ to $\mathscr{A}$, where $\mathscr{A}$ is a suitable abelian category, then various papers referred to below define equivalently the derived functors of $T$, whose value at $\Gamma \rightarrow \Pi$ we denote by $\mathfrak{B}_{n}(\Gamma, T)$. $\mathscr{A}$ will be either $\mathscr{M}_{\mathfrak{B}_{\Pi}}$ or $\boldsymbol{A b}$. None of these papers, except Stammbach [17], refers to varieties of groups but deals with more general situations, sometimes vastly more general. However, to avoid excessive proliferation, we leave the reader to make the necessary substitutions in these papers, and refer to the results and definitions they contain as if these substitutions had already been made, contenting ourself with a few comments on how this is to be done. Bachmann [2] refers to a functor $J: A \rightarrow B$. This becomes the embedding of $(\mathscr{P}, \Pi)$ in $(\mathfrak{B}, \Pi)$, which is also the interpretation we put on the functor $J: M \rightarrow C$ in Ulmer [19] and [20], and the embedding of $\boldsymbol{M}$ in $\boldsymbol{N}$ of André 
[1]. There is a difficulty in that $\mathscr{P}$ is not small, but this can be dealt with as in these papers. In Rinehart [13] interpret $\mathscr{E}$ as the class of surjections in $\mathfrak{B}$ and $\mathscr{P}$ as the class of $\mathscr{E}$-projectives in $\mathfrak{B}$, namely the splitting groups for $\mathfrak{B}$. In fact we may replace $\mathscr{P}$ by this possibly larger class in our interpretation of the above papers without changing the derived functors.

We now give a brief indication of the construction of the derived functors of $T$ starting with the triple theory of Barr and Beck, see [3], [4], and [5]. The obvious functor which takes a set $\mathfrak{x}$ to the $\mathfrak{B}$-free set on $\mathfrak{x}$ is left adjoint, by definition of freeness, to the forgetful functor from $\mathfrak{B}$ to sets. This induces a triple on the category of sets, and an algebra over this triple is just a group in $\mathfrak{B}$; in other words $\mathfrak{B}$ is triplable over sets. One now uses the induced cotriple in $\mathfrak{B}$ to construct the Barr-Beck resolution of a group $\Gamma$ in $\mathfrak{B}$. This is a simplicial resolution

$$
\longrightarrow \Gamma B_{n} \stackrel{\delta_{n}}{\longrightarrow} \Gamma B_{n-1} \longrightarrow \cdots \stackrel{\delta_{1}}{\longrightarrow} \Gamma B_{0} \stackrel{\varepsilon}{\longrightarrow} \Gamma
$$

of $\Gamma$, where for all $n \geqq 0, \Gamma B_{n}$ is $\mathfrak{B}$-free on the elements of the group to its right, $\delta_{n}$ is a certain ordered $n+1$-tuple of homomorphisms $\left(\delta_{n}^{0}, \ldots, \delta_{n}^{n}\right)$, and $\varepsilon$ is the canonical surjection. The $(n+1)$ ! homomorphisms of $\Gamma B_{n}$ to $\Gamma$ obtained by composing suitable homomorphisms coincide, so that if a homomorphism of $\Gamma$ into $\Pi$ is given, we may regard the above resolution as lying in $(\mathfrak{B}, \Pi)$. Alternatively one can use a normalised resolution in which each group is $\mathfrak{B}$-free on the nonidentity elements of the group to its right by using pointed sets instead of sets in the construction of the cotriple. One can construct much smaller resolutions by using "la construction pas à pas" of André [1] or the methods of Tierney and Vogel [18]; in fact only finitely generated groups will appear in the resolution provided that $\Gamma$ is finitely generated and the finitely generated groups of $\mathfrak{B}$ have the maximum condition on subgroups. But these resolutions are not functorial, and are more suited to specific calculations than to the general theory. If now $T$ is a functor from $\mathscr{P}$, or more generally from $(\mathscr{P}, \Pi)$, to the abelian category $\mathscr{A}$, then $\mathfrak{B}_{n}(\Gamma, T)$ is defined to be the $n$th homology group of

$$
\cdots \longrightarrow \Gamma B_{n} T \stackrel{d_{n}}{\longrightarrow} \Gamma B_{n-1} T \longrightarrow \stackrel{d_{1}}{\longrightarrow} \Gamma B_{0} T \longrightarrow 0
$$

where $d_{n}=\sum_{0}^{n}(-1)^{i} \delta_{n}^{i} T . \mathfrak{B}_{n}(-,-)$ thus defines a functor from $(\mathfrak{B}, \Pi) \times \mathscr{A}^{(\mathscr{P}, \Pi)}$ to $\mathscr{A}$ (ignoring a tiresome set-theoretic difficulty with $\mathscr{A}^{(\mathscr{P}, \Pi)}$ ). If $0 \rightarrow T_{1} \rightarrow T_{2}$ $\rightarrow T_{3} \rightarrow 0$ is a short exact sequence of functors from $(\mathscr{P}, \Pi)$ to $\mathscr{A}$, one obtains as usual a long exact sequence in homology, and $\mathfrak{B}_{n}(\Gamma, T)=0$ for $n>0$ if $T$ is projective in $\mathscr{A}^{(\mathscr{P}, \Pi)}$, that is to say if the Kan extension of $T$ (see below) is representable in the sense of Eilenberg and Mac Lane [7], see Ulmer [19]. Also, if $\Gamma$ is $\mathfrak{B}$-free, or more generally is a splitting group for $\mathfrak{B}$, then $\mathfrak{B}_{n}(\Gamma, T)=0$ for $n>0$ and any $T$. The cases which interest us most, though we shall be led to consider other functors, are $T=\operatorname{Diff}(-, A)$ and $T=\operatorname{Der}(-, A), A$ a fixed $\mathfrak{B} \Pi$-module; or rather the restriction to $(\mathscr{P}, \Pi)$ of these functors, which are defined on $(\mathfrak{B}, \Pi)$. Normally these functors 
take their values in $\boldsymbol{A} \boldsymbol{b}$ and $\boldsymbol{A} \boldsymbol{b}^{\text {op }}$ respectively, but we shall regard $\operatorname{Diff}(-, \mathfrak{B} \Pi)$ (and its derived functors) as taking values in $\mathscr{M} \mathfrak{B}_{\Pi}$. Note that Diff $(-, \mathfrak{B} \Pi)$ is the functor $D$ of the previous paragraph and that $\operatorname{Der}(-, A)=(\mathfrak{B}, \Pi)(-, A)$, where $A$ as always is confused with the split extension $A \Pi$. We write $\mathfrak{B}_{n}(\Gamma, A)$ and $\mathfrak{B}^{n}(\Gamma, A)$ for $\mathfrak{B}_{n}(\Gamma, \operatorname{Diff}(-, A))$ and $\mathfrak{B}^{n}(\Gamma, \operatorname{Der}(-, A))$ respectively. A short exact sequence of modules gives rise to a short exact sequence of functors from $(\mathscr{P}, \Pi)$ to $\boldsymbol{A b}$ or $\boldsymbol{A} \boldsymbol{b}^{\mathrm{op}}$, and hence to the usual long exact sequences in homology and cohomology. Moreover,

$$
\mathfrak{B}_{0}(\Gamma, A)=\operatorname{Diff}(\Gamma, A) \quad \text { and } \quad \mathfrak{B}^{0}(\Gamma, A)=\operatorname{Der}(\Gamma, A) .
$$

Observe that since the functor $\operatorname{Diff}(-, A)$ is the composite of the functors $\operatorname{Diff}(-, \mathfrak{B} \Pi)$ and $-\bigotimes_{\mathfrak{B} \Pi} A, \mathfrak{B}_{n}(\Gamma, A)$ is obtained by taking a complex of free $\mathfrak{B} \Pi$-modules over $D \Gamma$, tensoring with $A$, and taking homology. Thus $\mathfrak{B}_{n}(\Gamma,-)$ and $\operatorname{Tor}_{n}^{\mathfrak{B} \Pi}(D \Gamma,-)$ are equivalent functors for all $n$ if and only if $\mathfrak{B}_{n}(\Gamma, D \Pi)=0$ for all $n>0$. That this is the case for $\Pi=\Gamma$ and $\mathfrak{B}$ the variety of all groups is proved in [3]. We shall return to this problem for other varieties in the next paragraph and elsewhere, but let us observe here that if $A$ is a nonzero projective $\mathfrak{B} \Pi$-module, then $\operatorname{Diff}(-, A)$ does not define a projective object in $A b^{(\mathscr{P}, \Pi)}$. Finally, if $\alpha: \Gamma_{0} \rightarrow \Gamma_{1}$ is a surjection in $(\mathfrak{B}, \Pi)$, there is a long exact sequence

$$
\begin{aligned}
\cdots \rightarrow \mathfrak{B}_{n}\left(\Gamma_{0}, A\right) \rightarrow \mathfrak{B}_{n}\left(\Gamma_{1}, A\right) \rightarrow M_{n-1}(\alpha, A) \rightarrow \\
\quad \cdots \rightarrow M_{0}(\alpha, A) \rightarrow \mathfrak{V}_{0}\left(\Gamma_{0}, A\right) \rightarrow \mathfrak{B}_{0}\left(\Gamma_{1}, A\right) \rightarrow 0
\end{aligned}
$$

and similarly in cohomology which appears in Barr and Beck [4] as Proposition 2.2. The map of $\mathfrak{B}_{n}\left(\Gamma_{0}, A\right)$ into $\mathfrak{B}_{n}\left(\Gamma_{1}, A\right)$ is $\mathfrak{B}_{n}(\alpha, A)$.

We now turn to an alternative construction of the derived functors of $T . \mathscr{A}$ denotes $\boldsymbol{A b}$ or $\mathscr{M} \mathfrak{B}_{\Pi}$ or the dual of one of these categories. The fact that $\mathfrak{B}$ and $\mathscr{P}$ are not small will be ignored, a device justified in the various papers referred to below. For details the reader may refer to Bachmann [2], for example. The inclusion functor $J$ of $(\mathscr{P}, \Pi)$ into $(\mathfrak{B}, \Pi)$ induces a functor $J^{*}: \mathscr{A}^{(\mathfrak{B}, \Pi)} \rightarrow \mathscr{A}^{(\mathscr{P}, \Pi)}$, and this has a left adjoint $\tilde{J}: \mathscr{A}^{(\mathscr{P}, \Pi)} \rightarrow \mathscr{A}^{(\mathfrak{B}, \Pi)}$. Moreover, if $T:(\mathscr{P}, \Pi) \rightarrow \mathscr{A}$, then $\tilde{J} T$ is an extension of $T$, the Kan extension. $\tilde{J}$ is an additive functor from an abelian category with enough projectives into a second abelian category, and we define $\mathfrak{B}_{n}(-, T)$ to be the $n$th left derived functor of $\tilde{J}$ evaluated at $T$. It is clear that this is equivalent to Rinehart's construction in [13], and Ulmer [20] and Mitchell [11] prove that this is equivalent to André's construction in [1]. These papers also prove that this method is equivalent to the simplicial theory described above. Rinehart also constructs the exact sequence (2.1) [13, Theorem 2.18], and in the proof of Theorem 5.4, loc. cit., he identifies $M_{0}(\alpha, A)$, in the case $A=D \Pi$, and $\Gamma_{0} \rightarrow \Pi$ a surjection, as $R /[R, S]$ where $R=\operatorname{ker}\left(\Gamma_{0} \rightarrow \Gamma_{1}\right)$ and $S=\operatorname{ker}\left(\Gamma_{0} \rightarrow \Pi\right)$. Moreover the map $\gamma$ of $M_{0}(\alpha, D \Pi)$ into $\mathfrak{B}_{0}\left(\Gamma_{0}, D \Pi\right)=D \Gamma_{0}=I \Gamma_{0} \otimes_{z \Gamma_{0}} \mathfrak{B} \Pi$ is given by $r[R, S] \gamma$ $=(1-r) \otimes 1$. Of course Rinehart's argument refers to the variety of all groups, but the argument extends to the relative case without change. Now for $A$ a $\mathfrak{B} \Pi$ - 
module, $-\otimes_{\mathfrak{B} \Pi} A$ and $\mathscr{M}_{\Pi}(-, A)$ are right exact functors into $\boldsymbol{A b}$ and $\boldsymbol{A} \boldsymbol{b}^{\text {p }}$ respectively. It follows from Rinehart's definition of $M_{0}(\alpha, A)$ that this group and its homomorphism into $\mathfrak{B}_{0}\left(\Gamma_{0}, A\right)$ are obtained from the case $A=D \Pi$ by applying the functor $-\bigotimes_{\mathfrak{B} \Pi} A$, and similar remarks apply in cohomology. Summarizing, we have

THEOREM 2.1. Let

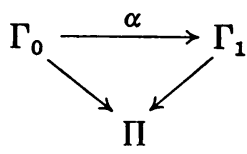

be surjections. Let $R=\operatorname{ker} \alpha, S=\operatorname{ker}\left(\Gamma_{0} \rightarrow \Pi\right)$, and $A$ be $a \mathfrak{B} \Pi$-module. Then there are exact sequences

$$
\begin{aligned}
& \mathfrak{B}_{1}\left(\Gamma_{0}, A\right) \longrightarrow \mathfrak{B}_{1}\left(\Gamma_{1}, A\right) \longrightarrow(R /[R, S]) \otimes_{\mathfrak{B} \Pi} A \\
& \stackrel{\gamma \otimes A}{\longrightarrow} \mathfrak{B}_{0}\left(\Gamma_{0}, A\right) \longrightarrow \mathfrak{B}_{0}\left(\Gamma_{1}, A\right) \longrightarrow 0
\end{aligned}
$$

and

$$
\begin{aligned}
& 0 \longrightarrow \mathfrak{B}^{0}\left(\Gamma_{1}, A\right) \longrightarrow \mathfrak{B}^{0}\left(\Gamma_{0}, A\right) \longrightarrow \mathscr{M}_{\mathfrak{B}_{\Pi}}(R /[R, S], A) \\
& \stackrel{\gamma \perp A}{\longrightarrow} \mathfrak{B}^{1}\left(\Gamma_{1}, A\right) \longrightarrow \mathfrak{B}^{1}\left(\Gamma_{0}, A\right)
\end{aligned}
$$

where the homomorphisms of $\mathfrak{B}_{i}\left(\Gamma_{0}, A\right)$ into $\mathfrak{B}_{i}\left(\Gamma_{1}, A\right), i=0,1$, are $\mathfrak{B}_{i}(\alpha, A)$ and similarly in cohomology. $\gamma \perp A$ denotes $\mathscr{M}_{\Pi}(\beta, A)$, and $\gamma$ is as above.

A special case of (2.2) appears in Stammbach [17]. See also Barr and Beck [4, (8.2)].

3. Nonisomorphism. We begin by stating the problem in general terms. Let $\mathscr{C}$ be a category with terminal object $\Pi$. We have in mind such categories as the comma category of groups, Lie algebras or restricted Lie algebras etc. over a fixed object $\Pi$. Consider the following conditions:

(i) $\mathscr{C}$ has enough projectives.

Using these projectives as models we will construct the derived functors $\mathscr{C}_{n}(-, T)$ of a functor $T$ from the full subcategory of projectives to a module category. In practice these projectives will be the image of a cotriple in $\mathscr{C}$, so we have the triple construction at our disposal.

(ii) The full subcategory of $\mathscr{C}$ formed by the abelian groups in $\mathscr{C}$ is (isomorphic to) $\mathscr{M}_{R}$, the category of right modules over a ring $R$.

(iii) The inclusion functor from $\mathscr{M}_{R}$ into $\mathscr{C}$ has a left adjoint $D$.

For $\Gamma \in|\mathscr{C}|$ and $A$ a left $R$-module, write $\operatorname{Diff}(\Gamma, A)$ for $D \Gamma \otimes_{R} A$ and $\mathscr{C}_{n}(-, A)$ for the $n$th derived functor of $\operatorname{Diff}(-, A)$. Similarly write $\mathscr{C}^{n}(-, A)$ for the $n$th derived functor of $\mathscr{C}(-, A), A$ a right $R$-module (or rather an abelian group in $\mathscr{C}$ ).

(iv) $\mathscr{C}_{n}(\Pi,-)$ and $\operatorname{Tor}_{n}^{R}(D \Pi,-)$ are equivalent functors from left $R$-modules to $\boldsymbol{A b}$. 
(v) $\mathscr{C}^{n}(\Pi,-)$ and $\operatorname{Ext}_{R}^{n}(D \Pi,-)$ are equivalent functors from right $R$-modules to $\boldsymbol{A b}$.

Note that in (iv) and (v) $\Pi$ should not be replaced by an arbitrary object $\Gamma$ of $\mathscr{C}$. In practice this greater generality can be achieved by making $R$ and $D$ depend on $\Gamma$. For example, in the category of groups over $\Pi, R=Z \Pi$; and in (iv) and (v) if $\Pi$ is replaced by $\Gamma, Z \Pi$ must be replaced by $Z \Gamma, A$ being a $\Gamma$-module by pullback. $D \Gamma$ is then the augmentation ideal of $\Gamma$.

Many of the standard algebraic categories satisfy these five conditions, see for example Rinehart [13] and [14]. We now restrict our attention to the case $\mathscr{C}=(\mathfrak{B}, \Pi)$, writing $\mathfrak{B}_{n}(\Pi, A)$ and $\mathfrak{B}^{n}(\Pi, A)$ for the appropriate (co-) homology groups as before. The functor $D$ was constructed in $\S 1$, and $R=\mathfrak{B} \Pi$. Conditions (i), (ii) and (iii) are already established. If $\mathfrak{B}$ is an abelian variety then $(\mathfrak{B}, \Pi)$ is an abelian category and (iv) and (v) follow from general theory. In fact if $\mathfrak{B}$ is the variety of abelian groups of exponent $m, m \geqq 0$, then $\mathfrak{B} \Pi=Z_{m}$, the ring of integers modulo $m$, and $D \Pi=\Pi$, so

$$
\mathfrak{B}_{n}(\Pi, A)=\operatorname{Tor}_{n}^{Z}(\Pi, A), \quad \mathfrak{B}^{n}(\Pi, A)=\operatorname{Ext}_{Z_{m}}^{n}(\Pi, A) .
$$

Of course these (co-) homology groups vanish for $n>1$ if $m=0$, and for $n>0$ if $m$ is square-free.

We now construct a situation in which (iv) and (v) fail. We start by constructing an analogue of a special case of Theorem 2.1.

LEMMA 3.1. Let $1 \rightarrow R \rightarrow F \stackrel{\alpha}{\rightarrow} \Pi \rightarrow 1$ be an exact sequence with $F \mathfrak{B}$-free. Let $K=\operatorname{ker}(D F \rightarrow D \Pi)$ and $A$ be a $\mathfrak{B} \Pi$-module. Then there are exact sequences

(3.2) $0 \rightarrow \operatorname{Der}(\Pi, A) \rightarrow \operatorname{Der}(F, A) \rightarrow \operatorname{Hom}_{\mathfrak{B} \Pi}(K, A) \rightarrow \operatorname{Ext}_{\mathfrak{B} \Pi}^{1}(D \Pi, A) \rightarrow 0$.

Proof. $0 \rightarrow K \rightarrow D F \rightarrow D \Pi \rightarrow 0$ is exact, and $D F$ is a free $\mathfrak{B} \Pi$-module. The given sequences are part of the long exact sequences one obtains from applying - $\otimes A$ or $\operatorname{Hom}(-, A)$.

If we now apply Theorem 2.1 in the case $\Gamma_{0}=F, \Gamma_{1}=\Pi$, we obtain, since $F$ is free, the sequences

$$
\begin{aligned}
0 & \rightarrow \mathfrak{V}_{1}(\Pi, A) \rightarrow\left(R / R^{\prime}\right) \otimes_{\mathfrak{B}_{\Pi}} A \rightarrow \operatorname{Diff}(F, A) \rightarrow \operatorname{Diff}(\Pi, A) \rightarrow 0, \\
0 & \rightarrow \operatorname{Der}(\Pi, A) \rightarrow \operatorname{Der}(F, A) \rightarrow \mathscr{M} \mathfrak{B}_{\Pi}\left(R / R^{\prime}, A\right) \rightarrow \mathfrak{B}_{1}(\Pi, A) \rightarrow 0 .
\end{aligned}
$$

Comparing (3.1) and (3.3) in the case $A=\mathfrak{B} \Pi$ gives, since $\operatorname{Tor}_{1}^{\mathfrak{B} \Pi}(D \Pi, \mathfrak{B} \Pi)=0$, a surjection $\beta$ of $R / R^{\prime}$ onto $K$.

LEMMA 3.2. With the hypotheses of Lemma 3.1 and $\beta$ as above, the following are equivalent:

(i) $\beta$ is an isomorphism;

(ii) $\mathfrak{B}_{1}(\Pi,-)$ and $\operatorname{Tor}_{1}^{\mathfrak{B} \Pi}(D \Pi,-)$ are equivalent functors from $\mathscr{M}_{\mathfrak{\Pi}}$ to $\boldsymbol{A b}$; 
(iii) $\mathfrak{B}_{1}(\Pi, \mathfrak{B} \Pi)=0$;

(iv) $\mathfrak{B}^{1}(\Pi,-)$ and $\operatorname{Ext}_{\mathfrak{B} \Pi}^{1}(D \Pi,-)$ are equivalent functors from $\mathscr{M}_{\mathfrak{B}_{\Pi}}$ to $\boldsymbol{A b}^{\mathrm{op}}$;

(v) $\mathfrak{B}^{1}(\Pi, J)=0$ for every injective $\mathfrak{B} \Pi$-module $J$.

In particular, (i) is independent of the choice of $F \stackrel{\alpha}{\rightarrow} \Pi$.

Proof. Using the homomorphism $\beta \otimes A:\left(R / R^{\prime}\right) \otimes A \rightarrow K \otimes A$, one obtains a mapping of the sequence (3.3) into the sequence (3.1). Hence (i) $\Rightarrow$ (ii). Clearly (ii) $\Rightarrow$ (iii) $\Rightarrow$ (i). Similarly (i) $\Rightarrow$ (iv) $\Rightarrow$ (v). Assuming (v), we prove (i). It is enough to prove that $\operatorname{Hom}(\beta, A): \operatorname{Hom}(K, A) \rightarrow \operatorname{Hom}\left(R / R^{\prime}, A\right)$ is an isomorphism for all $\mathfrak{B} \Pi$-modules $A$. Since $\operatorname{Hom}(K,-)$ and $\operatorname{Hom}\left(R / R^{\prime},-\right)$ are right exact, it is enough to check this for injective $\mathfrak{B} \Pi$-modules $J$. But since $\operatorname{Ext}_{\mathfrak{B} \Pi}^{1}(\Pi, J)$ $=0$, the result is clear.

We now need a practical device for calculating the homomorphism $\beta: R / R^{\prime} \rightarrow K$. If $\iota: K \rightarrow D F$ is the inclusion, then $\beta \iota=\gamma$, where $\gamma: R / R^{\prime} \rightarrow D F=I F \otimes_{z F} \mathfrak{B} \Pi$ is the homomorphism of the previous paragraph, namely $r R^{\prime} \gamma=(1-r) \otimes 1$. Suppose $F$ is $\mathfrak{B}$-free on the set $\mathfrak{x}$. The Fox derivatives are derivations of the absolutely free group on $\mathfrak{x}$ into its integral group ring. If $x \in \mathfrak{x}$, then $\partial / \partial x$ clearly induces a derivation, also denoted by $\partial / \partial x$, of $F$ into $\mathfrak{B} F$, and hence a derivation $\partial^{\alpha} / \partial x$ of $F$ into $\mathfrak{B} \Pi$. Now if $w \in F, 1-w=\sum\left(1-x_{i}\right) \partial w / \partial x_{i}$ summed over all $x_{i}$ in $\mathfrak{x}$. (The corresponding formula with left derivations for the variety of all groups appears in Fox [8].) By Lemma 1.2, $D F$ is the free $\mathfrak{B} \Pi$-module on generators $\left(1-x_{i}\right) \otimes 1$, $x_{i} \in \mathfrak{x}$. Putting all this together gives

LEMMA 3.3. Let $F$ be $\mathfrak{B}-$ free on the set $x$. Then $\beta: R / R^{\prime} \rightarrow K \subset D F=I F \otimes_{z F} \mathfrak{B} \Pi$ is given by

$$
r R^{\prime} \beta=\sum\left(1-x_{i}\right) \otimes \partial^{\alpha} r / \partial x_{i},
$$

the sum being taken over all $x_{i}$ in $\mathfrak{x}$.

We can now test the conditions of Lemma 3.2.

Proposition 3.4. If $\Pi$ is a cyclic group the conditions of Lemma 3.2 are satisfied.

Proof. If $\Pi$ is $\mathfrak{B}$-free, and in particular if $\Pi$ is absolutely free, then condition (iii) of Lemma 3.2 holds. Let $\Pi$ be a finite cyclic group generated by $a$, let $F$ be $\mathfrak{B}$-freely generated by $x$, and let $\alpha: F \rightarrow \Pi$ map $x$ to $a$. Suppose $r=x^{t}$ is in $\operatorname{ker} \beta$. By Lemma 3.3, $\partial^{\alpha} r / \partial x=0$, so $1+a+\cdots+a^{t-1}$ is in the ideal of $Z \Pi$ generated by images of the Fox derivatives of a set of defining words for $\mathfrak{B}$. We may take this set to consist of $x^{n}$ and a set of commutators, where $n$ is the characteristic of $\mathfrak{B}$ (Hanna Neumann [12]). If $n=0$ these derivatives are all in the augmentation ideal, so $t=0$. If $n>0$, then the derivatives are mapped by the augmentation to the multiples of $n$. Hence $t$ divides $n$, and $x^{t}$ represents the identity of $R$.

For a counterexample we thus need a noncyclic group and a nonabelian variety.

THEOREM 3.5. If $\Pi$ is Klein's four group, and $\mathfrak{B}=\mathfrak{R}_{2}$, the variety of groups nilpotent of class 2, then the conditions of Lemma 3.2 are not satisfied. 
Proof. Take $F$ to be $\mathfrak{B}$-free on $x$ and $y$, and $\alpha$ to map $x, y$ to generators $a, b$ of $\Pi$. Then $R$ is generated by $x^{2}, y^{2}$ and $[x, y]$, and $R^{\prime}$ is generated by $\left[x^{2}, y^{2}\right]=[x, y]^{4}$. Hence $[x, y]^{2} R^{\prime}$ is a nontrivial element of $R / R^{\prime}$ which we prove is in the kernel of $\beta$. By Lemma 3.3, this is equivalent to proving that

$$
\partial^{\alpha}[x, y]^{2} / \partial x=\partial^{\alpha}[x, y]^{2} / \partial y=0 .
$$

A simple calculation shows that $\partial^{\alpha}[x, y]^{2} / \partial x=2(b-1)$. Using Proposition 1.3, we want to show that $2(b-1)$ is in $(I \Pi)^{2}$. But since $b^{2}=1,-(b-1)^{2}=2(b-1)$. Similarly $\partial^{\alpha}[x, y]^{2} / \partial y=0$.

It follows that the classical theory of Tor and Ext is of no use to us, and we are forced to rely on the ideas outlined in the previous paragraph. In particular the technique of dimension shifting is denied us.

4. The relative multiplier. We begin with a universal coefficient lemma; for a special case, see Stammbach [17]. If $A$ is a $\Pi$-module with trivial $\Pi$-action, then clearly $A$ is a $\mathfrak{B} \Pi$-module if and only if $A$ lies in $\mathfrak{B}$ qua group, i.e. if and only if the exponent of $A$ divides the exponent of $\mathfrak{B}$. In particular, if $\mathfrak{B}$ is of exponent $m$, and if $Z_{m}$ denotes $Z / m Z$, with trivial $\Pi$-action, then $Z_{m}$ is a $\mathfrak{B} \Pi$-module.

LEMMA 4.1. If $\mathfrak{B}$ is of exponent $m$ and $A$ is a $\mathfrak{B} \Pi$-module with trivial $\Pi$-action, then there are split exact sequences

$$
\begin{aligned}
0 & \rightarrow \mathfrak{V}_{n}\left(\Pi, Z_{m}\right) \otimes A \rightarrow \mathfrak{B}_{n}(\Pi, A) \rightarrow \operatorname{Tor}_{1}^{Z_{m}}\left(\mathfrak{B}_{n-1}\left(\Pi, Z_{m}\right), A\right) \rightarrow 0, \\
0 & \rightarrow \operatorname{Ext}_{Z_{m}}^{1}\left(\mathfrak{B}_{n-1}\left(\Pi, Z_{m}\right), A\right) \rightarrow \mathfrak{B}^{n}(\Pi, A) \rightarrow \operatorname{Hom}\left(\mathfrak{B}_{n}\left(\Pi, Z_{m}\right), A\right) \rightarrow 0
\end{aligned}
$$

if $m=0$, or if $m$ is square-free, or if $A=Z_{m}$. In the last two cases the Tor and Ext terms vanish.

Proof. Using a simplicial resolution of $\Pi$, one obtains $\mathfrak{B}_{*}\left(\Pi, Z_{m}\right)$ as the homology of a complex of free $Z_{m}$-modules. $\mathfrak{B}_{*}(\Pi, A)$ and $\mathfrak{B}^{*}(\Pi, A)$ are obtained from the same complex after tensoring with or homming into $A$. This gives the result (Cartan and Eilenberg [6, Theorems VI 3.3 and VI 3.3a]).

The Schur multiplier of a finite group $\Pi$ may be defined in any of the following equivalent ways (see Schur [15], [16]). $1 \rightarrow R \rightarrow F \rightarrow \Pi \rightarrow 1$ is exact, $F$ a free group:

(1) $H_{2}(\Pi, Z)$

(2) $H^{2}(\Pi, Q / Z)$;

(3) $R \cap F^{\prime} /[R, F]$;

(4) the largest group $M$ for which there is an extension $0 \rightarrow M \rightarrow E \rightarrow \Pi \rightarrow 1$, with $M \subseteq(\zeta E) \cap E^{\prime}$.

In this case call $E \rightarrow \Pi$ a covering of $\Pi$ and $E$ a covering group of $\Pi$. Note in particular that (3) is independent of the presentation given, and that (4) is meaningful, $M$ being finite. Every covering of $\Pi$ (up to the obvious equivalence) can be constructed as follows. Take $F$ to be finitely generated. The sequence

$$
0 \rightarrow R \cap F^{\prime} /[R, F] \rightarrow R /[R, F] \rightarrow F / F^{\prime}
$$


induced by inclusions is exact. $F / F^{\prime}$ is a free abelian group and $M$ (say) $=R \cap F^{\prime} /[R, F]$ is finite since $H_{2}(\Pi, Z)$ is. It follows that $R \cap F^{\prime} /[R, F]$ is the torsion subgroup of the finitely generated group $R /[R, F]=T$ (say). Let $S$ be a maximal torsion-free subgroup of $T$, so that $T=S \oplus M$. Put $H=F /[R, F]$. Then $0 \rightarrow M \rightarrow H / S \rightarrow \Pi \rightarrow 1$ is a covering of $\Pi$, and any covering of $\Pi$ (up to equivalence) may be obtained in this way for a suitable choice of $S$. Finally, if $0 \rightarrow A$ $\rightarrow \Gamma \rightarrow \Pi \rightarrow 1$ is any exact sequence with $A \subseteq \zeta \Gamma \cap \Gamma^{\prime}$, then there is a covering $E \rightarrow \Pi$ of $\Pi$ and a surjection of $E$ onto $\Gamma$ such that the obvious diagram commutes. We now indicate how all this can be relativised.

THEOREM 4.2. If $\Pi$ is finite, $\mathfrak{B}$ is of exponent zero, and $1 \rightarrow \bar{R} \rightarrow \bar{F} \rightarrow \Pi \rightarrow 1$ is exact with $\bar{F} \mathfrak{B}$-free, then the following groups are isomorphic:

(1) $\mathfrak{B}_{1}(\Pi, Z)$;

(2) $\mathfrak{B}^{1}(\Pi, Q / Z)$;

(3) $\bar{R} \cap \bar{F}^{\prime} /[\bar{R}, \bar{F}]$;

(4) the largest group $\bar{M}$ for which there is an extension $0 \rightarrow \bar{M} \rightarrow E \rightarrow \Pi \rightarrow 1$ with $\bar{M} \subseteq(\zeta E) \cap E^{\prime}$ and $E$ in $\mathfrak{B}$.

In this case call $E \rightarrow \Pi$ a $\mathfrak{B}$-covering of $\Pi, E$ a $\mathfrak{B}$-covering group of $\Pi$, and $\bar{M}$ the $\mathfrak{B}$-multiplier of $\Pi$. In particular, (3) is independent of the $\mathfrak{B}$-presentation given, and (4) is meaningful.

Proof. (1) and (2) are isomorphic by universal coefficients. To prove that (1) and (3) are isomorphic consider the exact sequence

$$
0 \rightarrow \mathfrak{B}_{1}(\Pi, Z) \rightarrow \bar{R} /[\bar{R}, \bar{F}] \rightarrow \bar{F} / \bar{F}^{\prime}
$$

obtained either from putting $\Gamma_{0}=\bar{F}, \Gamma_{1}=\Pi, \Pi=$ the trivial group, and $A=Z$ in (2.2) or $A=Z, F=\bar{F}$ in (3.3).

Putting $\bar{M}=\bar{R} \cap \bar{F}^{\prime} /[\bar{R}, \bar{F}]$, we prove that it has the property (4). We may take $\bar{F}$ to be $\mathfrak{B}$-free on a finite set $\mathfrak{x}$. Taking $F$ to be absolutely free on $\mathfrak{x}$, construct a commutative diagram

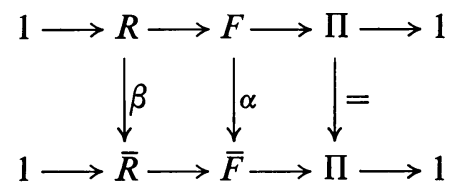

where $\alpha$ is the natural surjection. This induces a commutative diagram

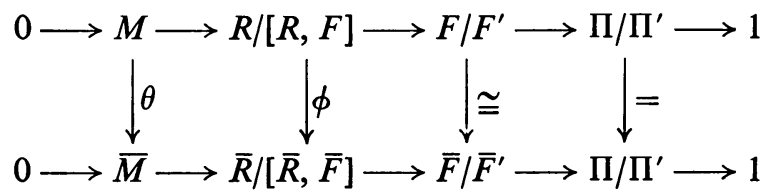

where $M=R \cap F^{\prime} /[R, F]$. The map of $F / F^{\prime}$ onto $\bar{F} / \bar{F}^{\prime}$ is an isomorphism since $\mathfrak{B}$ is of exponent zero. $\theta$ and $\phi$ are surjections. Let $R /[R, F]=S \oplus M$. Then $S$ is a 
free abelian group, and since $\phi$ has finite kernel, $\phi$ induces an isomorphism of $S$ onto a subgroup $\bar{S}$ of $\bar{R} /[\bar{R}, \bar{F}]$. Clearly $\bar{R} /[\bar{R}, \bar{F}]=\bar{S} \oplus \bar{M}$, and, since $\bar{R} /[\bar{R}, \bar{F}]$ is in the centre of $\bar{F} /[\bar{R}, \bar{F}], \bar{S}$ is normal in $\bar{F} /[\bar{R}, \bar{F}]=\bar{H}$, say. Now $0 \rightarrow \bar{M} \rightarrow \bar{H} / \bar{S}$ $\rightarrow \Pi \rightarrow 1$ is clearly an extension of $\bar{M}$ by $\Pi$. Also, $\bar{H} / \bar{S}=G$, say, lies in $\mathfrak{B}$ and $\bar{M} \subseteq(\zeta G) \cap G^{\prime}$.

It remains to prove that if $0 \rightarrow A \rightarrow E \rightarrow \Pi \rightarrow 1$ is any extension of $A$ by $\Pi$ with $E$ in $\mathfrak{B}$ and $A \subseteq(\zeta E) \cap E^{\prime}$, then $E$ is a homomorphic image of $\bar{H} / \bar{S}$ for some choice of $S$. Since $E$ is already a homomorphic image of $H / S$ for some $S$, it is enough to prove that the homomorphism $\psi$ of $H / S$ into $\bar{H} / \bar{S}$ induced by $\alpha$ is the surjection obtained by dividing out by the $\mathfrak{B}$ words in $H / S$. We have a commutative diagram

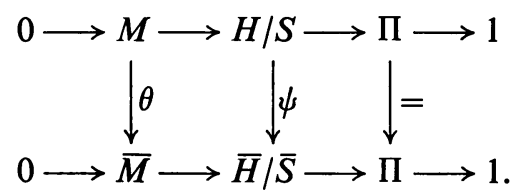

Since $\theta$ is a surjection, so is $\psi$. It remains to prove that every element of the kernel of $\psi$ is in the verbal subgroup of $H / S$ determined by $\mathfrak{B}$. Let $\omega$ be in the kernel of $\psi$. Then $\omega$ is the image of an element $r[R, F]$ of the kernel of

$$
\theta: R \cap F^{\prime} /[R, F]=M \rightarrow \bar{M}=\bar{R} \cap \bar{F}^{\prime} /[\bar{R}, \bar{F}] .
$$

Since $\alpha$ maps $[R, F]$ onto $[\bar{R}, \bar{F}], r$ may be taken to lie in the kernel of $\alpha$. Then $r$ lies in the verbal subgroup of $F$ determined by $\mathfrak{B}$. But $H / S=(F /[R, F]) / S$ is a quotient group of $F$, and $M \rightarrow H / S$ is induced by the inclusion. So $\omega$, being represented by $r$, is in the required subgroup of $H / S$. This completes the proof of the theorem.

We have only needed the full force of our hypotheses in the last part of the theorem.

Proposition 4.3. Let $\mathfrak{B}$ be of exponent $m$ and $\Pi$ be any group in $\mathfrak{B}$. If $1 \rightarrow \bar{R}$ $\rightarrow \bar{F} \rightarrow \Pi \rightarrow 1$ is exact with $F \mathfrak{B}-$ free, then the following are isomorphic:

(1)' $\mathfrak{B}_{1}\left(\Pi, Z_{m}\right)$;

(3) $)^{\prime} \bar{R} \cap \bar{F}^{\prime} /[\bar{R}, \bar{F}]$;

moreover, if $\Pi$ is finite, these are isomorphic to

(2)' $\mathfrak{B}^{1}\left(\Pi, Z_{m}\right)$.

Proof. Since $Z_{m}$ is an injective $Z_{m}$-module, the proof of the corresponding parts of the theorem need only slight adjustment.

As we shall see later the restriction to varieties of exponent zero is essential for the last part of the theorem, at least in

LEMMA 4.4. Let $\mathfrak{B} \subseteq \mathfrak{W}$ be varieties of exponent zero, and $\Pi$ be a finite group in $\mathfrak{B}$. If $E$ is a $\mathfrak{W}$-covering group of $\Pi$, then the homomorphic image of $E$ obtained by dividing out by the verbal subgroup of $E$ determined by $\mathfrak{B}$ is a $\mathfrak{B}$-covering group of $\Pi$; and 
conversely every $\mathfrak{B}$-covering group of $\Pi$ may be obtained in this way from some W-covering group.

Proof. It is enough to consider the case when $\mathfrak{W}$ is the variety of all groups, when the result follows at once from the proof of Theorem 4.2.

We can now establish a sufficient condition for two finite groups to generate the same variety. If $\Pi$ is any group we denote by varo $\Pi$ the variety generated by $\Pi$ and the infinite cyclic group. Thus varo $\Pi$ is the least variety of exponent zero which contains $\Pi$.

THEOREM 4.5. Let $\Pi$ be a finite group in the variety $\mathfrak{B}$ of exponent zero. Let $E_{1}, E_{2}$ be $\mathfrak{B}$-covering groups of $\Pi$. Then varo $E_{1}=$ varo $E_{2}$.

Proof. It is enough, by symmetry, to prove that $E_{2}$ is in varo $E_{1}$. Suppose that it is not. Apply Lemma 4.4 with $\mathfrak{B}, \mathfrak{B}$ and $E$ replaced by varo $E_{1}, \mathfrak{B}$ and $E_{1}$ respectively. We see that $E_{1}$ is a varo $E_{1}$-covering of $\Pi$. Now apply the lemma with $\mathfrak{B}, \mathfrak{W}$ and $E$ replaced by varo $E_{1}, \mathfrak{B}$ and $E_{2}$ respectively. We see that a proper homomorphic image of $E_{2}$ is a varo $E_{1}$-covering of $\Pi$. But since any two covering groups of $\Pi$ with respect to the same variety must have the same order, we have a contradiction.

COROLlARY 4.6. Let $E_{1}, E_{2}$ be $\mathfrak{B}$-covering groups of $\Pi$, of exponent $m, n$ respectively. Let $\mathfrak{w}$ be the set of all commutator laws of var $E_{1}$. Then $x^{n}$ and $\mathfrak{w}$ define var $E_{2}$.

Proof. This is just a rewording of the theorem.

The theorem and corollary are of most interest when $\mathfrak{B}$ is the variety of all groups. For example one obtains the well-known fact (Hanna Neumann [12]) that the quaternion and dihedral groups of order 8 generate the same variety, being covering groups of Klein's four group. Again, the nonabelian groups of order $p^{3}$ are covering groups of the elementary abelian group of order $p^{2}$, but one of these groups is of exponent $p$ and the other of exponent $p^{2}$, so the restriction to varieties of exponent zero in Theorem 4.2, or at least in Lemma 4.4, is needed.

We now show by a simple example that the concept of covering group is essential to Theorem 4.5 in the sense that there is a finite group $\Pi$, a group $A$, and extensions $E_{1}, E_{2}$ of $A$ with $A \subseteq\left(\zeta E_{i}\right) \cap E_{i}^{\prime}, i=1,2$, but varo $E_{1} \neq$ varo $E_{2} . D_{n}$ denotes the dihedral group of order $n$.

$$
\begin{gathered}
\Pi=D_{8} \times C_{2}=\left\langle x, y, z \mid x^{2}=y^{2}=[x, y]^{2}=z^{2}=[x, z]=[y, z]=1\right\rangle, \\
E_{1}=D_{16} \times C_{2}=\left\langle a, b, c \mid a^{2}=b^{2}=[a, b]^{4}=c^{2}=[a, c]=[b, c]=1\right\rangle,
\end{gathered}
$$

$E_{2}$ is the split extension of $K=\left\langle p, q \mid p^{2}=q^{2}=(p q)^{2}=1\right\rangle$ by

$$
D_{8}=\left\langle r, s \mid r^{2}=s^{2}=[r, s]^{2}=1\right\rangle
$$

where $r$ centralizes $K$, and $p^{s}=p, q^{s}=p q$.

Now define $\alpha_{1}: E_{1} \rightarrow \Pi$ by $a \alpha_{1}=x, b \alpha_{1}=y, c \alpha_{1}=z$; and define $\alpha_{2}: E_{2} \rightarrow \Pi$ by $r \alpha_{2}=x, s \alpha_{2}=y, p \alpha_{2}=1, q \alpha_{2}=z$. Then $\operatorname{ker} \alpha_{1}=\left\langle[a, b]^{2}\right\rangle$ and $\operatorname{ker} \alpha_{2}=\langle p\rangle=\langle[q, s]\rangle$; 
so in each case $E_{i}$ is an extension of a 2-cycle in $\left(\zeta E_{i}\right) \cap E_{i}^{\prime}$ by $\Pi$. But $\zeta E_{2}$ contains $(r s)^{2}$ and $p$, so $E_{2} / \zeta E_{2}$ is elementary abelian, whereas $E_{1}$ is nilpotent of class 3 . Hence, varo $E_{1} \neq$ varo $E_{2}$.

5. Products. We produce an analogue for the Künneth formula in our dimension one, basing the proof on that of Schur [16] for finite groups in the universal variety. One would not expect to be able to do much in higher dimensions since our dimension $n$ corresponds to the classical dimension $n+1$; and in fact we shall show in a later paper that strange things happen in our dimension 2.

If $F$ is a $\mathfrak{B}$-free group then the exponent of $F_{(2)} / F_{(3)}$ is, as we see below, independent of the rank of $F$ provided this rank is greater than one. This exponent we call the derived exponent of $\mathfrak{B}$. In discussing the derived exponent we may assume that $\mathfrak{B}$ is nilpotent of class 2 . In this case $\mathfrak{B}$ is defined by at most three laws, $x^{e}, \prod_{i=1}^{r}\left[x_{\alpha i}, x_{\beta i}\right]^{n_{i}}$, and $[x, y, z]$; where the $r$ unordered pairs $\{\alpha i, \beta i\}$ are distinct. The second of these laws is clearly equivalent to $[x, y]^{m}$ where $m$ is the h.c.f. of $\left\{n_{1}, \ldots, n_{r}\right\}$. Moreover if $a, b$ are elements of any nilpotent group of class 2 then $(a b)^{n}=a^{n} b^{n}[a, b]^{n(n-1) / 2}$. Using these observations it is easy to prove

LEMMA 5.1. The derived exponent of a variety $\mathfrak{B}$ is the h.c.f. of $k$ and $l$ where $k$ is the exponent of $\mathfrak{B}$ if this is odd and half the exponent if this is even, and lis obtained as follows: Take any set $\mathfrak{w}$ of laws in $x_{1}, x_{2}, \ldots$ which define $\mathfrak{B}$. We may assume that each $w$ in $\mathfrak{w}$ consists of a power of $x_{1}$, followed by powers $\left[x_{i}, x_{j}\right]^{w_{i j}}$ of distinct commutators $\left[x_{i}, x_{j}\right]$ with $i<j$, followed by commutators of greater weight. Then $l$ is the h.c.f. of $\left\{w_{i j} \mid w \in \mathfrak{w}, i<j\right\}$.

THEOREM 5.2. Let $\mathfrak{B}$ have exponent $m$ and derived exponent $t$; and let $\Pi_{1}, \Pi_{2}$ be any groups in $\mathfrak{B}$. Then

$$
\mathfrak{B}_{1}\left(\Pi_{1} \times \Pi_{2}, Z_{m}\right)=\mathfrak{B}_{1}\left(\Pi_{1}, Z_{m}\right) \oplus \mathfrak{B}_{1}\left(\Pi_{2}, Z_{m}\right) \oplus\left(\Pi_{1} / \Pi_{1}^{\prime} \otimes_{z} \Pi_{2} / \Pi_{2}^{\prime} \otimes_{z} Z_{t}\right) .
$$

Note that, if $\mathfrak{B}$ is abelian, then $t=1$ (but not conversely) and the result is obvious.

Proof. Let $1 \rightarrow R_{i} \rightarrow F_{i} \rightarrow \Pi_{i} \rightarrow 1$ be exact with $F_{i} \mathfrak{B}$-free, $i=1,2$. Then we have $1 \rightarrow R \rightarrow F \rightarrow \Pi_{1} \times \Pi_{2} \rightarrow 1$ exact, where $F$ is the verbal product of $F_{1}$ and $F_{2}$, and $R$ is the subgroup generated by the images of $R_{1}, R_{2}$, and $\left[F_{1}, F_{2}\right]$. Since any element of $F$ is uniquely of the form $w_{1} w_{2} c, w_{i} \in F_{i}, c \in\left[F_{1}, F_{2}\right]$ (Hanna Neumann [12]), it follows that $R \cap F^{\prime} /[R, F]$ is isomorphic to the direct sum of $R_{1} \cap F_{1}^{\prime} /\left[R_{1}, F_{1}\right], R_{2} \cap F_{2}^{\prime} /\left[R_{2}, F_{2}\right]$, and the subgroup generated by $\left[F_{1}, F_{2}\right][R, F]$. By Proposition 4.3 it remains to evaluate this last group. We may take $F_{1}, F_{2}$ to be $\mathfrak{B}$-free on $\left\{x_{a} \mid a \in \Pi_{1}\right\}$ and $\left\{y_{u} \mid u \in \Pi_{2}\right\}$ respectively, with the obvious surjections onto $\Pi_{1}$ and $\Pi_{2}$. Then $\left[F_{1}, F_{2}\right] /\left(\left[F_{1}, F_{2}\right] \cap F_{(3)}\right)$ is the free $Z_{t}$-module on $\left\{\left[x_{a}, y_{u}\right] \mid a \in \Pi_{1}, u \in \Pi_{2}\right\}$. A quick calculation shows that $\left[F_{1}, F_{2}\right] \cap F_{(3)}$ is a normal subgroup of $\left[F_{1}, F_{2}\right] \cap[R, F]$, and that

$$
\left(\left[F_{1}, F_{2}\right] \cap[R, F]\right) /\left(\left[F_{1}, F_{2}\right] \cap F_{(3)}\right)
$$

is generated by the cosets containing $\left\{\left[x_{a} x_{b} x_{c}^{-1}, y_{u}\right] \mid a b=c\right\}$ and

$$
\left\{\left[x_{a}, y_{u} y_{v} y_{w}^{-1}\right] \mid u v=w\right\} \text {. }
$$


Thus $\left[F_{1}, F_{2}\right] /\left(\left[F_{1}, F_{2}\right] \cap[R, F]\right)$ is the free $Z_{t}$-module on $\left\{\left[x_{a}, y_{u}\right]\right\}$ with these relations, namely $\Pi_{1} / \Pi_{1}^{\prime} \otimes_{z} \Pi_{2} / \Pi_{2}^{\prime} \otimes_{z} Z_{t}$.

Added in proof. I am grateful to Peter M. Neumann for showing me a grouptheoretical proof of Theorem 4.5.

\section{BIBLIOGRAPHY}

1. M. André, Méthode simpliciale en algèbre homologique et algèbre commutative, Lecture Notes in Math., no. 32, Springer-Verlag, Berlin and New York, 1967. MR 35 \#5493.

2. F. Bachmann, Kategorische Homologietheorie und Spectralsequenzen, Math. Report \#17, Battelle Institute, 1969 (mimeograph).

3. M. Barr and J. Beck, Acyclic models and triples, Proc. Conference Categorical Algebra (La Jolla, Calif., 1965), Springer, New York, 1966, pp. 336-343. MR 39 \#6955.

4. - Homology and standard constructions, Sem. on Triplets and Categorical Homology Theory (ETH, Zürich, 1966/67), Springer, Berlin, 1969, pp. 245-335. MR 41 \#3562.

5. J. Beck, Triples and cohomology, Thesis, Columbia University, New York, 1965.

6. H. Cartan and S. Eilenberg, Homological algebra, Princeton Univ. Press, Princeton, N. J., 1956. MR 17, 1040.

7. S. Eilenberg and S. Mac Lane, Acyclic models, Amer. J. Math. 75 (1953), 189-199. MR 14, 670 .

8. R. H. Fox, Free differential calculus. I, Ann. of Math. (2) 57 (1953), 547-560. MR 14, 843.

9. M. Hall, The theory of groups, Macmillan, New York, 1959. MR 21 \#1996.

10. J. Knopfmacher, Extensions in varieties of groups and algebras, Acta Math. 115 (1966), 17-50. MR 35 \#4287.

11. B. Mitchell, Rings with several objects, Dalhousie University, 1970 (mimeograph).

12. Hanna Neumann, Varieties of groups, Springer-Verlag, New York, 1967. MR 35 \#6734.

13. G. S. Rinehart, Satellites and cohomology, J. Algebra 12 (1969), 295-329. MR 39 \#6953.

14. ——, Errata, J. Algebra 14 (1970), 125-126. MR 40 \#1455.

15. I. Schur, Uber die Darstellung der endlichen Gruppen durch gebrochene lineare Substitutionen, Crelle 127 (1904), 20-50.

16. — Untersuchungen über die Darstellung der endlichen Gruppen durch gebrochene lineare Substitutionen, Crelle 132 (1907), 85-137.

17. U. Stammbach, Homological methods in group varieties, Comment. Math. Helv. 45 (1970), 287-298.

18. M. Tierney and W. Vogel, Simplicial derived functors, Category Theory, Homology Theory and their Applications. I, Battelle Institute Conference (Seattle, Wash., 1968), vol. 1, Springer, Berlin, 1969, pp. 167-180. MR 39 \#4249.

19. F. Ulmer, Acyclic models and Kan extensions, Category Theory, Homology Theory and their Applications. I, Battelle Institute Conference (Seattle, Wash., 1968), vol. 1, Springer, Berlin, 1969, pp. 181-204. MR 41 \#1840a.

20. - Kan extensions, cotriples and André (co) homology, Category Theory, Homology Theory and their Applications. II, Battelle Institute Conference (Seattle, Wash., 1968), vol. 2, Springer, Berlin, 1969, pp. 278-308. MR 41 \#1840b.

Queen Mary College, London, England 